\title{
LOCAL PROPERTIES OF SECANT VARIETIES IN SYMMETRIC PRODUCTS. PART II
}

\author{
TRYGVE JOHNSEN
}

\begin{abstract}
Let $V$ be a linear system on a curve $C$. In Part I we described a method for studying the secant varieties $V_{d}^{r}$ locally. The varieties $V_{d}^{r}$ are contained in the $d$-fold symmetric product $C^{(d)}$.

In this paper (Part II) we apply the methods from Part I. We give a formula for local tangent space dimensions of the varieties $V_{d}^{1}$ valid in all characteristics (Theorem 2.4).

Assume rk $V=n+1$ and $\operatorname{char} K=0$. In $\S \S 3$ and 4 we describe in detail the projectivized tangent cones of the varieties $V_{n}^{1}$ for a large class of points. The description is a generalization of earlier work on trisecants for a space curve.

In $\S 5$ we study the curve in $C^{(2)}$ consisting of divisors $D$ such that $2 D \in$ $V_{4}^{1}$. We give multiplicity formulas for all points on this curve in $C^{(2)}$ in terms of local geometrical invariants of $C$. We assume $\operatorname{char} K=0$.
\end{abstract}

\section{INTRODUCTION}

Let $C$ be a nonsingular curve over a field $K$, and let $V \subset H^{0}(C, L)$ be a linear system on $C$, where $L$ is a line bundle. Denote by $C^{(d)}$ the $d$ th symmetric product of $C$. The subschemes $V_{d}^{r}$ of $C^{(d)}$ consist of those divisors that impose at most $d-r$ independent conditions on $V$. The $V_{d}^{r}$ are secant varieties.

As an example consider the case where rk $V=4$ and $V$ is very ample. Then $V$ defines an embedding of $C$ into $P^{3}$. The sections of $V$ are then thought of as hyperplanes of $P^{3}$. The variety $V_{3}^{1}$ parametrizes those divisors of degree 3 that consist of 3 collinear points on $C$ in $P^{3}$. Roughly speaking: $V_{3}^{1}$ parametrizes the trisecant lines of the embedded curve.

It is a well-known fact that the $V_{d}^{r}$ can be defined scheme-theoretically as the zero schemes

$$
Z\left(\bigwedge^{d-r+1} \sigma\right) \text { for } r=1, \ldots, d
$$

where $\sigma$ is a canonical $C^{(d)}$-bundle map

$$
\sigma: V \otimes \mathscr{O}_{C^{(d)}} \rightarrow E_{L},
$$

Received by the editors September 10, 1987.

1980 Mathematics Subject Classification (1985 Revision). Primary 14M15, 14H45, $14 \mathrm{~B} 12$.

Key words and phrases. Secant varieties of curves, local geometry. 
and $E_{L}$ is a vector bundle of rank $d$ on $C^{(d)}$ obtained from $L$ by a so-called symmetrization process. See, for example, [ACGH, p. 340].

In Part I we constructed a computational device for studying the map $\sigma$ and the varieties $V_{d}^{r}$ locally. Our main results were given in Theorem 4.2 and Proposition 4.4 of Part I. We constructed a local matrix description of $\sigma$ and described the formal completion $\hat{O}_{V_{d}^{r}, D}$ of the local ring of $V_{d}^{r}$ at a point (divisor) $D$. Such a local description is often trivial when $D$ consists of $d$ distinct points. The main purpose with our results is to study the $V_{d}^{r}$ at points on the diagonal in $C^{(d)}$. In Part II we will use the information from Part I to obtain some geometrical results.

In $\S 2$ we give a formula for the tangent space dimension of the variety $V_{d}^{1}$ at a point $D$. The formula is valid in any characteristic.

In $\S 3$ we study a large class of points on the variety $V_{n}^{1}$, where rk $V=n+1$. We describe the tangent cones of $V_{n}^{1}$ at such points, and in particular we give a formula for the multiplicity of $V_{n}^{1}$ at these points.

In $\S 4$ we find further properties of the tangent cones described in $\S 3$. We will indicate when the projectivized tangent cones are singular. This is a generalization of a result in $[\mathrm{J}]$ concerning trisecant lines for a space curve.

In $\S 5$ we study stationary bisecants for a nonsingular space curve. A stationary bisecant is a bisecant line, where the curve tangents at the points of secancy meet, or a tangent line at a point where the osculating plane of the curve is hyperosculating. We define a curve in $C^{(2)}$ that parametrizes these situations, and we describe the local structure of this curve. We find out how the tangent cone of the curve in $C^{(2)}$ at a secant divisor is determined by the local geometry of $C$ at the points of secancy. The study of stationary bisecants was proposed to the author by Ignacio Sols. Similar results to those of our $\S 5$ have been found independently by Miguel Gonzalez [G]. He uses bundles of principal parts. Global properties of the varieties $V_{d}^{r}$ and of the various diagonals in $C^{(d)}$ are described in [ACGH, Chapter VIII], in the case $K=\mathbf{C}$. Our paper can be read as a local supplement of that chapter.

Remark. References to results and formulas in Part I will be marked by the prefix I. Theorem I.4.2. means Theorem 4.2. of Part I, equation I.(4.1) means equation (4.1) of Part I and so on.

\section{The tANGENT SPACE DIMENSION of $V_{d}^{1}$ AT $D \in C^{(d)}$}

The varieties $V_{d}^{1}$ are interesting since they parametrize divisors that are "special" with respect to the linear systems $V$.

Let $D=\sum_{i=1}^{k} d_{i} P_{i}$, where $D \in V_{d}^{1}$, and the $P_{i}$ are distinct points on $C$. We will use Theorem I.4.2. to compute the tangent space dimension of $V_{d}^{1}$ at $D$. The Brill-Noether matrix $B N$ (see I.(4.2)) consists of $k$ groups of consecutive rows, where the $i$ th group (consisting of $d_{i}$ rows) corresponds to the point $P_{i}$, for $i=1, \ldots, k$. 
Definition 2.1. $l_{i}$ is the maximal integer $s \in\left\{0, \ldots, d_{i}-1\right\}$ such that the matrix consisting of all rows of $B N$ except the $(s+1)$ st row in the $i$ th group has rank $d-1$. If no such integer exists, set $l_{i}=-1$.

Explanation. Assume for simplicity that $V$ is base-point free and thus maps $C$ into some $P^{n}$. For a chosen set of local parameters of $C$ at the $P_{i}$ we can talk about derivative vectors of $C$ at the $P_{i}$. Call the point $P_{i}$ itself the 0 th derivative vector of $C$ at $P_{i}$. Then $l_{i}$ is the maximal integer $s \in\left\{0, \ldots, d_{i}-1\right\}$ such that the union of the 0 th $, \ldots, \hat{s}$ th $, \ldots,\left(d_{i}-1\right)$ st derivative vectors of $C$ at $P_{i}$ and the 0 th, $\ldots,\left(d_{j}-1\right)$ st derivative vectors at $P_{j}$, for $j \neq i$, span a $d-2$ plane in $P^{n}$. If no such $s$ exists, then $l_{i}=-1$.

Observation 2.2. $D \in V_{d}^{2} \Leftrightarrow l_{i}=-1$ for $i=1, \ldots, k$.

Definition 2.3. Assume $D^{\prime} \in C^{\left(d^{\prime}\right)}$ for some $d^{\prime} \in N$. Denote by $V\left(-D^{\prime}\right)$ the linear system $V \cap H^{0}\left(C, L\left(-D^{\prime}\right)\right)$. We now give the main result of this section (valid in any characteristic).

Theorem 2.4. The tangent space dimension of $V_{d}^{1}$ at $D$ is

$$
\min \left(d, \mathrm{rk} V\left(-\sum_{i=1}^{k}\left(d_{i}+l_{i}+1\right) P_{i}\right)+2 d-n-2\right), \text { where rk } V=n+1
$$

Proof. It is enough to study the constant and linear parts of the matrix $M$ (I.(4.1)). Since rk $B N \leq d-1$, we may assume that only the $d-1$ first columns of $B N$ are nonzero. Since we will only study the linear parts of the $d$-minors, we may assume that the entries in the $d-1$ first columns are constant. Assume first $D=d P$. We may drop the index $i$ in $M$, and we have

$$
\begin{aligned}
& M=\left[\begin{array}{ccccc}
a_{0,0} & \ldots & a_{d-2,0} & (-1)^{d-1} a_{d-1, d} s_{d} & \ldots \\
a_{0,1} & \ldots & a_{d-2,1} & (-1)^{d-2} a_{d-1, d} s_{d-1}+(-1)^{d-1} a_{d-1, d+1} s_{d} & \ldots \\
\vdots & & \vdots & \vdots & \\
a_{0, d-1} & \ldots & a_{d-2, d-1} & a_{d-1, d} s_{1}+\cdots+(-1)^{d-1} a_{d-1,2 d-1} s_{d} & \ldots
\end{array}\right. \\
& \left.\begin{array}{l}
(-1)^{d-1} a_{n, d} s_{d} \\
(-1)^{d-2} a_{n, d} s_{d-1}+(-1)^{d-1} a_{n, d+1} s_{d} \\
\vdots \\
a_{n, d} s_{1}+\cdots+(-1)^{d-1} a_{n, 2 d-1} s_{d}
\end{array}\right] .
\end{aligned}
$$

Here we used that the linear part of $W_{j}\left(s_{1}, \ldots, s_{d}\right)$ is $(-1)^{j-1} s_{j}$ for $j=$ $1, \ldots, d$. See I.(3.4). Let $D_{j-1}$ be the $d-1$ minor formed by the $d-1$ first columns of $M$ (or $B N$ ) minus the $j$ th row. We see that $l$ is the largest integer $j$ such that $D_{j} \neq 0$ if such an integer exists (see Definition 2.1).

The linear parts of the $n+2-d$ relations cutting out $V_{d}^{1}$ are (up to signs):

$$
\begin{gathered}
\left(a_{i, d} D_{l}\right) s_{d-l}-\left(a_{i, d+1} D_{l}+a_{i, d} D_{l-1}\right) s_{d-l+1}+\cdots \\
+(-1)^{l}\left(a_{i, d+l} D_{l}+\cdots+a_{i, d} D_{0}\right) s_{d}
\end{gathered}
$$

for $i=d-1, \ldots, n$. 
The coefficient matrix of these relations in $s_{1}, \ldots, s_{d}$ is easily seen to have the same rank as

$$
N=\left[\begin{array}{ccc}
a_{d-1, d} & \ldots & a_{d-1, d+l} \\
\vdots & & \vdots \\
a_{n, d} & \ldots & \alpha_{n, d+l}
\end{array}\right]
$$

Hence the tangent space dimension of $V_{d}^{1}$ at $D$ is $d-\operatorname{rk} N$ if $l \geq 0$ and $d$ otherwise. Assume first $l \geq 0$. Let us find

$$
\text { rk } V(-(l+d+1) P) \text {. }
$$

Since $l \geq 0$, Observation 2.2. gives that the matrix $B N$ has rank exactly $d-1$, and therefore a section contained in $V(-d P)$ must be of the form $c_{d-1} X_{d-1}+$ $\cdots+c_{n} X_{n}$, where the $c_{j} \in K$, and where $X_{j}$ is the section corresponding to the $(j+1)$ st column of $M$. The conditions that such a section should be contained in $V(-(l+d+1) P)$ are

$$
\begin{array}{ccc}
a_{d-1, d} c_{d-1} & +\cdots+ & a_{n, d} c_{n}=0, \\
\vdots & & \vdots \\
a_{d-1, d+l} c_{d-1} & +\cdots+ & a_{n, d+l} c_{n}
\end{array}
$$

These equations in the variables $c_{d-1}, \ldots, c_{n}$ give rise to a coefficient matrix, which is the transpose of $N$.

Hence rk $V(-(l+d+1) P)=n-d+2-\operatorname{rk} N$, and we deduce that the tangent space dimension of $V_{d}^{1}$ at $D$ is

$$
d-\operatorname{rk} N=2 d-n-2+\operatorname{rk} V(-(l+d+1) P) .
$$

Since rk $V(-(l+d+1) P) \leq \mathrm{rk} V(-d P)=n-d+2$, our tangent space dimension is at most $(2 d-n-2)+(n-d+2)=d$. Hence the theorem holds when $D=d P$, and $l \geq 0$.

When $D=d P$ and $l=-1$, the tangent space dimension is $d$ since all the $D_{j}$ are zero. On the other hand,

$$
\begin{gathered}
2 d-n-2+\mathrm{rk} V(-(l+d+1) P)=2 d-n-2+\mathrm{rk} V(-d P) \\
=(2 d-n-2)+(n+1-\mathrm{rk} B N) \geq d+1
\end{gathered}
$$

since $\mathrm{rk} B N \leq d-2$. Hence $d$ is the minimum of $d$ and $2 d-n-2+$ rk $V(-(l+d+1) P)$. Our proof is now complete in the case $D=d P$. The general case follows easily using the same argument for each group of $d_{i}$ rows of $M$.

\section{A LOCAL STUdy OF $V_{n}^{1}$ WHERE rk $V=n+1 \geq 4$}

In [ $\mathrm{J}$, Theorem 2.3.1], we gave a multiplicity formula for trisecant lines to a space curve. In this section we will generalize this formula.

Let $D \in C^{(n)}$ be a point of $V_{n}^{1}$, where rk $V=n+1 \geq 4$. Assume:

(1) for each $D^{\prime} \in C^{(n-1)}$, such that $D^{\prime} \leq D$, we have $D^{\prime} \notin V_{n-1}^{1}$; 
(2) if $D=\sum_{i=1}^{k} n_{i} P_{i}$ (all $\left.n_{i}>0\right)$, then $D+P_{i} \notin V_{n+1}^{2}$, for $i=1, \ldots, k$;

(3) $\operatorname{char} K=0$ and $K=\bar{K}$.

Proposition 3.1. Under assumptions (1), (2) and (3), we have:

(a) the tangent space dimension of $V_{n}^{1}$ at $D$ is $\mathrm{rk} V(-2 D)+n-2$, where rk $V(-2 D)$ is 0 or 1 ;

(b) $\operatorname{dim} O_{V_{n}^{1}, D}=n-2$;

(c) the multiplicity of $V_{n}^{1}$ at $D$ is the largest integer $s$ such that $\mathrm{rk} V(-s D) \geq$ 1 (with equality if $V_{n}^{1}$ is singular at $D$ ).

Proof. Let $l_{i}$, for $i=1, \ldots, k$, be the integers described in Definition 2.1 . Assumption (1) gives $l_{i}=n_{i}-1$ for all $i$. The tangent space dimension formula in (a) is then a special case of Theorem 2.4, and it holds also when $\operatorname{char} K>0$.

Assumption (2) gives that $\mathrm{rk} V(-2 D)$ is 0 or 1 , because if rk $V(-2 D) \geq 2$, then $2 D \in V_{2 n}^{n+1}$, and then $D+P_{i} \in V_{n+1}^{2}$ for all $i \in\{1, \ldots, k\}$. Hence (a) holds.

By general facts about determinantial varieties, we have $\operatorname{dim} O_{V_{n}^{1}, D} \geq n-2$.

If $\mathrm{rk} V(-2 D)=0$, then the tangent space dimension of $V_{n}^{1}$ at $D$ is $n-2$ by (a). Hence, $\operatorname{dim} \hat{O}_{V_{n}^{1}, D} \leq n-2$, and (b) follows. Furthermore $V_{n}^{1}$ is nonsingular at $D$ in this case. Hence the multiplicity of $V_{n}^{1}$ at $D$ is 1 . Since rk $V(-2 D)=$ 0 , and $\operatorname{rk} V(-1 \cdot D) \geq 2 \geq 1$, the number given in (c) is also 1 . Hence (c) follows when $\mathrm{rk} V(-2 D)=0$.

It remains to prove (b) and (c) when rk $V(-2 D)=1$. Let $V$ be generated by the sections $\left\{X_{0}, \ldots, X_{n}\right\}$. rk $V(-D) \geq 2$ since $D \in V_{n}^{1}$, and rk $V(-D) \leq 2$ since $D \notin V_{n}^{2}$ by (1).

Hence rk $V(-D)=2$, and we may assume that $X_{n-1}$ and $X_{n}$ generate $V(-D)$. This means that the entries in the two last columns of the $B N$ matrix (see I.(4.2)) are zero.

We may assume that $X_{n}$ generates $V(-2 D)$ since rk $V(-2 D)=1$. The matrix $M$ of I.(4.1) will have constant terms only in the entries of the $n-1$ first columns, corresponding to $X_{0}, \ldots, X_{n-2}$ (since $X_{n-1}, X_{n}$ are in $V(-D)$ ). On the other hand, each of these $n-1$ columns will have at least one entry with a nonzero constant term. This implies that the ideal generated by the $n$-minors of $M$ is, in fact, generated by the two $n$-minors obtained by disregarding each of the two last columns, corresponding to $X_{n-1}$ and $X_{n}$. We call these minors $R_{n-1}$ and $R_{n}$, respectively.

The strategy now is as follows: (1) and (2) imply that the leading form of $R_{n}$ is linear in the variables $s_{1,1}, \ldots, s_{k, n_{k}}$. One uses $R_{n}$ to express one of the $s_{i, j}$, say $s_{i 0, j 0}$, as a power series in the remaining $s_{i, j}$ modulo the ideal $\left(R_{n}\right)$ in $K[[\underline{s}]]$. Then one substitutes this power series for $s_{i 0, j 0}$ in $R_{n-1}$ to get a new relation $\bar{R}_{n-1}$ in the remaining $s_{i, j}$. Using I.(3.4) it is easy to show that 
the leading form of $R_{n-1}$ is of degree $m$, where $m$ is

$$
\max \{s \mid \mathrm{rk} V(-s D)=1\} \geq 2 \text {. }
$$

It is less obvious, but still true, that the leading form of $\bar{R}_{n-1}$ is of degree $m$ also. The verification of this is done in detail in [Prpr] for the case $D=n P$. There we also indicate how this verification can be modified to apply to the general case $D=\sum_{i=1}^{k} n_{i} P_{i}$. This gives the conclusion of our proposition.

Definition 3.2. For a variety $X$ and a point $P$ in $X$ the tangent cone $\mathscr{T}_{P}(X)$ of $X$ at $P$ is

$$
\operatorname{Spec}\left(\bigoplus_{i=0}^{\infty} m^{i} / m^{i+1}\right) \text {, }
$$

where $m$ is the maximal ideal of the local ring $O_{X, P}$.

The projectivized tangent cone $P \mathscr{T}_{P}(X)$ of $X$ at $P$ is

$$
\operatorname{Proj}\left(\bigoplus_{i=0}^{\infty} m^{i} / m^{i+1}\right) \text {. }
$$

Corollary 3.3. Under (1), (2) and (3), the projectivized tangent cone $P \mathscr{T}_{D}\left(V_{n}^{1}\right)$ is a hypersurface of degree $m$ in $P^{n-2}$, where

$$
m=\max \{s \mid \mathrm{rk} V(-s D) \geq 1\} .
$$

Proof. Corollary 3.3 follows from the proof of Proposition 3.1 .

4. The tANGENT CONE $\mathscr{T}_{D}\left(V_{n}^{1}\right)$, Where rk $V=n+1 \geq 4$

In this section we will not always prove our assertions. Our goal is to give a geometrical interpretation of $\mathscr{T}_{D}\left(V_{n}^{1}\right)$ (or $\left.P \mathscr{T}_{D}\left(V_{n}^{1}\right)\right)$ described at the end of $\S 3$.

In $\S 3$ we studied a point $D$ in $V_{n}^{1}$, where $\operatorname{rk} V=n+1 \geq 4$. Under (1), (2) and (3) of $\S 3$ we gave a description of the dimension, embedding dimension and multiplicity of $V_{n}^{1}$ at $D$.

A question which then arises naturally is: When is the projectivized tangent cone $P \mathscr{T}_{D}\left(V_{n}^{1}\right)$ singular? If $n=3$ and $V_{n}^{1}$ is a curve, then $P \mathscr{T}_{D}\left(V_{n}^{1}\right)$ is singular if $V_{n}^{1}$ does not have normal crossings at $D$; we also say that $V_{n}^{1}$ possesses a nonordinary singularity at $D$ in this case. In $[\mathrm{J}]$ we gave necessary and sufficient local conditions on $C$ for determining whether the trisecant curve (essentially $V_{3}^{1}$ ) possesses nonordinary singularities or not. We want to generalize these conditions to apply to any $V_{n}^{1}, n \geq 3$, where $\operatorname{rk} V=n+1$. In order to do this we assume:

$V$ is base-point free and $D+P \notin V_{n+1}^{2}$ for any point $P \in C$.

Assumption $\left(2^{\prime}\right)$ is, of course, a strengthening of $(2)$ of $\S 3$; but this strengthening is of no importance for the local geometry of $V_{n}^{1}$ at $D$. Whatever local 
result we prove for $V_{n}^{1}$ at $D$ under (1), $\left(2^{\prime}\right)$ and (3) will also hold under Assumptions (1), (2) and (3). This is true because the matrix $M$ (see I.(4.1)) is only dependent on the behaviour of $V$ at the points $P_{1}, \ldots, P_{k}$, and because any base point of $V$ is outside $\left\{P_{1}, \ldots, P_{k}\right\}$ by (1).

Under $\left(2^{\prime}\right) \quad V$ defines a map $\phi: C \rightarrow \bar{C} \subset P^{n}$. Let $G=G(n-2, n)$ be the Grassmannian, which parametrizes the $n-2$ planes in $P^{n}$.

For an $n-2$ plane $H$ denote by $[H]$ the corresponding point in $G$. Denote by $F$ the incidence variety

$$
\left\{([H], P) \in G \times P^{n} \mid P \in H\right\} .
$$

Consider the following diagram:

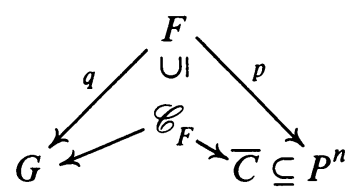

Here $p$ and $q$ are the natural projection maps from $F$ to $P^{n}$ and $G$, respectively, and $\mathscr{C}_{F}=p^{-1}(\bar{C})$.

Let Sec be the subvariety of $G$ cut out by the sheaf of $O_{G}$-ideals: $F^{n-1}\left(q_{*} O_{\mathscr{C}_{F}}\right)$, that is, the sheaf of $(n-1)$ st. Fitting ideals of the $O_{G}$-sheaf $q_{*} O_{\mathscr{E}_{F}}$. Then Sec parametrizes $n-2$ planes that are $n$-secant to $C$. This definition of Sec is taken from [GP], where the case $n=3$ is treated. As in $\S 3$ set $D=\sum n_{i} P_{i}$, where $\sum n_{i}=n$.

Assume $D \in V_{n}^{1}$, and that (1), $\left(2^{\prime}\right)$ and (3) hold. Then $D$ spans a unique $n-2$ plane; that is, $P_{1}, \ldots, P_{k}$ and the $n_{i}-1$ first derivative vectors of $\bar{C}$ at $P_{i}$ for $i=1, \ldots, k$, span a unique $n-2$ plane $H$.

We make the following claim:

$$
\mathscr{T}_{D}\left(V_{n}^{1}\right) \simeq \mathscr{T}_{[H]}(\mathrm{Sec})
$$

In fact, we strongly believe

$$
\hat{O}_{V_{n}^{1}, D} \simeq \hat{O}_{\mathrm{Sec},[H]} .
$$

We have not made any attempts to prove (4.2), but we have proved (4.1) when $D$ consists of $n$ distinct points.

To find $\mathscr{T}_{D}\left(V_{n}^{1}\right)$ one simply calculates the leading forms of the relations $R_{n-1}(\underline{s})$ and $R_{n}(\underline{s})$ described in the proof of Proposition 3.1. In [J] an explicit description of $\mathscr{T}_{[H]}(\mathrm{Sec})$ is given in the case where $n=3$, whether $D$ consists of three distinct points or not.

It is easy, but a little painstaking, to generalize this explicit description to arbitrary $n \geq 3$, when the $n$ points of $D$ are distinct. Comparing the two tangent cones one sees that they are isomorphic.

We omit the very technical calculations here. In principle the same method should work when the $n$ points are not distinct. 
Definition 4.1. For a curve $C$ and a hypersurface $M$ in $P^{n}$, denote by $I(P, C \cap M)$ the usual intersection number between $C$ and $M$ at $P$.

In the following we will only use (4.1) in Corollary 4.4. Assume that (1), $\left(2^{\prime}\right)$ and (3) hold for $D$. From Proposition 3.1 (a) and from local results about Sec we have: Sec is singular at $[H] \Leftrightarrow V_{n}^{1}$ is singular at $D \Leftrightarrow$ There exists a unique hyperplane $\mathscr{H}$ in $P^{n}$ with

$$
I\left(P_{i}, \bar{C} \cap \mathscr{H}\right) \geq 2 n_{i} \text { for } i=1, \ldots, k .
$$

We have Sec $\subset G \subset P^{S}$ for some large $S$. Making explicit calculations analogous to those in [GP and J] one finds that the embedded (compactified) tangent space in $P^{S}$ to Sec at $[H]$ is $\check{\mathscr{H}} \subset G \subset P^{S}$, where $\ddot{\mathscr{H}}$ is the $n-1$ plane in $G$, which parametrizes the $n-2$ planes in the hyperplane $\mathscr{H} \subset P^{n}$.

Hence the embedded tangent cone in $P^{S}$ to Sec at $[H]$ is a union of an $(n-3)$-dimensional family of lines in $\mathscr{\mathscr { H }}$. Each point of the projectivized tangent cone $P \mathscr{T}_{[H]}(\mathrm{Sec})$ or $P \mathscr{T}_{D}\left(V_{n}^{1}\right)$ corresponds to one such line.

A line $L$ in $\mathscr{\mathscr { H }}$ through $[H]$ is a nesting of a one-dimensional family of $n-2$ planes in $\mathscr{H}$ containing a fixed $n-3$ plane $h_{L}$ contained in $H$.

Hence each point of $P \mathscr{T}_{[H]}(\mathrm{Sec})$ and $P \mathscr{T}_{D}\left(V_{n}^{1}\right)$ corresponds to an $n-3$ plane $h_{L}$ in the $n-2$ plane $H$. Denote by $[h]$ the point in $\check{H}$ corresponding to an $n-3$ plane $h$, where $\check{H}$ is the $n-2$ plane which parametrizes the $n-3$ planes in $H$.

By Corollary 3.3, $P \mathscr{T}_{D}\left(V_{n}^{1}\right)$ is a hypersurface of degree

$$
m=\max \{s \mid \mathrm{rk} V(-s D) \geq 1\}
$$

in $P^{n-2}$. From the above discussion it is clear that a natural geometrical interpretation of this $P^{n-2}$ is $\breve{H}$, and that $P \mathscr{T}_{[H]}(\mathrm{Sec}) \cong\left\{\left[h_{L}\right] \mid L\right.$ is a line in $\check{\mathscr{H}}$ through $[H]$, such that

$L$ is contained in the embedded tangent cone to Sec at $[H]\}$.

Two problems now arise in a natural way:

(i) Find those $n-3$ planes $h$ in $H$ such that $[h] \in P \mathscr{T}_{[H]}(\mathrm{Sec})$.

(ii) Find those $n-3$ planes $h$ in $H$ such that [h] is a singular point of $P \mathscr{T}_{[H]}(\mathrm{Sec})$.

We state without proofs the solutions to problems (i) and (ii) (Results 4.2 and 4.3, respectively). Result 4.2 is a generalization of Theorem 2.3.2 of [J], and Result 4.3 is a generalization of Theorem 2.3.3 in [J].

We have proved Results 4.2 and 4.3 in the case where $D$ consists of $n$ distinct points, but we omit the technical details here.

Result 4.2. Under (1), $\left(2^{\prime}\right)$ and (3) we have $[h] \in P \mathscr{T}_{[H]}(\mathrm{Sec})$ if and only if there exists a hypersurface $M$ in $P^{n}$ such that:

(a) $\operatorname{deg} M=m+1$, and $M$ has a singularity of multiplicity at least $m$ at all points of $h$; 
(b) $I\left(P_{i}, M \cap \bar{C}\right) \geq(m+1) n_{i}$ for all $P_{i} \in H \cap C$;

(c) $m \cdot H \subseteq M \cap \mathscr{H}$, i.e. $I(M) \subseteq\left(I(\mathscr{H})+I(H)^{m}\right)$, and $H \nsubseteq \operatorname{Sing}(M)$;

(d) the equation defining $M$ in $P^{n}$ is equal to the equation of a cone of degree $m+1$ with $h$ contained in its vertex set, modulo the square of the ideal defining $H$.

Remark. $M$ can be taken to be a union of a one-dimensional family of $n-2$ planes containing $H$. Thus $M$ gives rise to a curve [ in $G$. The tangent line to [ at $[H]$ is $L$, where $h=h_{L}$.

Result 4.3. Under (1), $\left(2^{\prime}\right)$ and (3) we have: $[h]$ is a singular point of $P \mathscr{T}_{[H]}(\mathrm{Sec})$ if and only if there exists a hypersurface $N$ in $P^{n}$ such that:

(a) $N$ is a cone of degree $m$, and $h$ is contained in the vertex set of $N$;

(b) $I\left(P_{i}, \bar{C} \cap N\right) \geq(m+1) n_{i}$ for $i=1, \ldots, k$;

(c) $H \nsubseteq \operatorname{Sing}(N)$.

Corollary 4.4. Assume that (1), (2), (3), and (4.1) hold for D. We have: $P \mathscr{T}_{D}\left(V_{n}^{1}\right)$ is singular if and only if there exists a cone $N$ and an $n-3$ plane $h$ as described in Result 4.3(a), (b), (c).

\section{Stationary bisecants For A SPACE CURVE}

In $\S 5$ we assume char $K=0$, and $K=\bar{K}$. Let $C$ be a nonsingular curve in $P^{3}$, and let $P_{1}$ and $P_{2}$ be points on $C$. The line $\overline{P_{1} P_{2}}$ is usually called a stationary bisecant if the tangents to $C$ at $P_{1}$ and $P_{2}$ meet. In general there is a one-dimensional family of stationary bisecants for a space curve. We will define a scheme in $C^{(2)}$, which essentially parametrizes divisors $P_{1}+P_{2}$ with $P_{1}$ and $P_{2}$ as described. Some divisors $2 P$ may also occur as points on this scheme in $C^{(2)}$ since tangent lines are in some sense bisecants.

Let $C$ be mapped into $P^{3}$ by evaluating sections of some linear system $V$ of rank 4. Consider the map:

$$
i: C^{(2)} \rightarrow C^{(4)}, \quad \text { where } i(D)=2 D
$$

for divisors $D$ in $C^{(2)}$.

Definition 5.1. The scheme of stationary bisecants for $C$ with respect to $V$ is $i^{-1}\left(V_{4}^{1}\right)$.

Remark 5.2. Clearly $D \in i^{-1}\left(V_{4}^{1}\right) \Leftrightarrow 2 D \in V_{4}^{1}$. If $P_{1} \neq P_{2}$, then $P_{1}+P_{2} \in$ $i^{-1}\left(V_{4}^{1}\right) \Leftrightarrow$ the tangent lines to $C$ at $P_{1}$ and $P_{2}$ meet.

We also have:

$2 P \in i^{-1}\left(V_{4}^{1}\right) \Leftrightarrow P$ is a flex on $C$, or the osculating plane of $C$ at $P$ is hyperosculating.

It will follow from the proofs of Propositions 5.3 and 5.5 that $i^{-1}\left(V_{4}^{1}\right)$ is either a curve or empty. 
We will use Theorem I.4.2. to determine the multiplicity of $i^{-1}\left(V_{4}^{1}\right)$ at an arbitrary point $D$ (in $C^{(2)}$ ) in terms of the local geometry of $C$ at the secant points in $P^{3}$. The cases $D=2 P$ and $D=P_{1}+P_{2} \quad\left(P_{1} \neq P_{2}\right)$ will be treated separately. As before we denote by $I(Q, C \cap F)$ the intersection multiplicity between a curve $C$ and a surface $F$ at a point $Q$ in $P^{3}$.

The multiplicity of the curve $i^{-1}\left(V_{4}^{1}\right)$ at $D=P_{1}+P_{2}$. Assume $P_{1} \neq P_{2}$, and let $L$ be the line $\overline{P_{1} P_{2}}$. Set $n_{i}=I\left(P_{i}, C \cap H\right)$ for $i=1,2$, where $H$ is a general member of the pencil of planes containing $L$. We may assume $n_{1} \geq n_{2}$.

Let $r$ be the maximal integer such that there exists a plane $H$ with

$$
I\left(P_{i}, C \cap H\right) \geq n_{i}+r \quad \text { for } i=1,2 .
$$

Let $r_{2}$ be the maximal integer such that there exists a plane $H_{2}$ containing $L$ with

$$
I\left(P_{2}, C \cap H_{2}\right)=n_{2}+r_{2} .
$$

Proposition 5.3. The multiplicity of the curve $i^{-1}\left(V_{4}^{1}\right)$ at $P_{1}+P_{2}$ is

$$
\min \left(n_{1}+n_{2}+r-2,2 n_{2}+r_{2}-1\right) \text {. }
$$

Proof. Choose coordinates $X_{0}, X_{1}, X_{2}, X_{3}$ for $P^{3}$, and let $t_{i}$ be a local parameter at $P_{i}$ for $i=1,2$. Without loss of generality we choose

$$
\begin{aligned}
& X_{0}=1, \\
& X_{1}=t_{i}+k_{i}, \\
& X_{2}=\sum_{j \geq n_{i}} \alpha_{i, j} t_{i}^{j}, \\
& X_{3}=\sum_{j \geq n_{i}+r} \beta_{i, j} t_{i}^{j}
\end{aligned}
$$

as local parametrizations at $P_{i}$ for $i=1,2$.

By the definitions of $n_{1}, n_{2}$, and $r$, we may assume that $\alpha_{i, n_{1}}$ and $\alpha_{2, n_{2}}$ are nonzero, and that $\beta_{1, n_{1}+r}$ or $\beta_{2, n_{2}+r}$ is nonzero.

We see that the line $L=\overline{P_{1} P_{2}}$ has equations $X_{2}=X_{3}=0$, and that $P_{i}=$ $\left(1, k_{i}, 0,0\right)$ for $i=1,2$, with $k_{1} \neq k_{2}$.

The unique plane (if any) that intersects $C$ at least $n_{i}+1$ times at $P_{i}$ for $i=1,2$, has equation $X_{3}=0$. This is also the equation of $H_{2}$.

By Theorem I.4.2, we have

$$
\hat{O}_{V_{4}^{1}, 2 P_{1}+2 P_{2}} \cong K\left[\left[s_{1,1}, s_{1,2}, s_{2,1}, s_{2,2}\right]\right] /(\operatorname{det} M),
$$

where

$$
M=\left[\begin{array}{cccc}
1 & k_{1}+s_{1,1} & \sum_{j \geq n_{1}} \alpha_{1, j} W_{j}\left(s_{1,1}, s_{1,2}\right) & \sum_{j \geq n_{1}+r} \beta_{1, j} W_{j}\left(s_{1,1}, s_{1,2}\right) \\
0 & 1 & \sum_{j \geq n_{1}} \alpha_{1, j} W_{j-1}\left(s_{1,1}, s_{1,2}\right) & \sum_{j \geq n_{1}+r} \beta_{1, j} W_{j-1}\left(s_{1,1}, s_{1,2}\right) \\
1 & k_{2}+s_{2,1} & \sum_{j \geq n_{2}} \alpha_{2, j} W_{j}\left(s_{2,1}, s_{2,2}\right) & \sum_{j \geq n_{2}+r} \beta_{2, j} W_{j}\left(s_{2,1}, s_{2,2}\right) \\
0 & 1 & \sum_{j \geq n_{2}} \alpha_{2, j} W_{j-1}\left(s_{2,1}, s_{2,2}\right) & \sum_{j \geq n_{2}+r} \beta_{2, j} W_{j-1}\left(s_{2,1}, s_{2,2}\right)
\end{array}\right] .
$$


The map $i: C^{(2)} \rightarrow C^{(4)}$, where $i(D)=2 D$ induces a map

$$
i^{*}: \hat{O}_{C^{(4)}, 2 P_{1}+2 P_{2}} \rightarrow \hat{O}_{C^{(2)}, P_{1}+P_{2}} \text {. }
$$

Now

$$
\begin{aligned}
\hat{O}_{C^{(4)}, 2 P_{1}+2 P_{2}} & \simeq \hat{O}_{C^{(2)}, 2 P_{1}} \underset{K}{\otimes} \hat{O}_{C^{(2)}, 2 P_{2}} \\
& \simeq K\left[\left[s_{1,1}, s_{1,2}, s_{2,1}, s_{2,2}\right]\right],
\end{aligned}
$$

where the $s_{l, j}$ can be regarded as formal, algebraically independent, variables.

$s_{l, j}$ can also be regarded as the $j$ th elementary function in two replicas $t_{l, 1}, t_{l, 2}$ of the local parameter $t_{l}$ of $C$ at $P_{l}$ for $l=1,2, j=1,2$.

Furthermore,

$$
\hat{O}_{C^{(2)}, P_{1}+P_{2}} \simeq \hat{O}_{C, P_{1}} \underset{K}{\otimes} \hat{O}_{C, P_{2}} \simeq K\left[\left[t_{1}, t_{2}\right]\right]
$$

Hence we regard $i^{*}$ as a map

$$
i^{*}: K\left[\left[s_{1,1}, s_{1,2}, s_{2,1}, s_{2,2}\right]\right] \rightarrow K\left[\left[t_{1}, t_{2}\right]\right] .
$$

We have $\hat{O}_{i^{-1}\left(V_{4}^{1}\right), P_{1}+P_{2}} \simeq K\left[\left[t_{1}, t_{2}\right]\right] / \operatorname{det} M\left(i^{*} s_{1,1}, \ldots, i^{*} s_{2,2}\right)$. Clearly $i^{*} s_{l, j}=$ $s_{l, j}\left(t_{l}, t_{l}\right), \quad l=1,2, j=1,2$. From I.(3.5) we then obtain

$$
i^{*} W_{j}\left(t_{l}\right)=W_{j}\left(i^{*} s_{l, 1}, i^{*} s_{l, 2}\right)=(j+1) t_{l}^{j} .
$$

This implies that

$$
\hat{O}_{i^{-1}\left(V_{4}^{1}\right), P_{1}+P_{2}} \simeq K\left[\left[t_{1}, t_{2}\right]\right] /(R),
$$

where $R$ is the determinant of the matrix obtained from $M$ by substituting $W_{j}\left(s_{l, 1}, s_{l, 2}\right)$ by $(j+1) t_{l}^{j}$ for $l=1,2, j \geq 0$.

Calculation gives that the leading form of $R$ is

$$
\left(k_{1}-k_{2}\right)\left[n_{1}\left(n_{2}+r\right) \alpha_{1, n_{1}} \beta_{2, n_{2}+r} t_{2}^{r}-n_{2}\left(n_{1}+r\right) \alpha_{2, n_{2}} \beta_{1, n_{1}+r} t_{1}^{r}\right] \cdot t_{1}^{n_{1}-1} \cdot t_{2}^{n_{2}-1}
$$

or

$$
r_{2} \alpha_{2, n_{2}} \beta_{2, n_{2}+r_{2}} \cdot t_{2}^{2 n_{2}+r_{2}-1}
$$

or the sum of these forms.

One must check that neither of the forms vanishes identically as a polynomial in $t_{1}, t_{2}$, and that the forms do not cancel each other. Clearly (5.3) does not vanish. (5.3) cancels (5.2) only if $n_{1}=1$, but then $n_{2}=1$ also, and the forms have different degrees. Hence they do not cancel each other. For the form (5.2) we have two cases:

(a) $r=0$. Then the form vanishes iff

$$
\alpha_{1, n_{1}} \beta_{2, n_{2}}-\alpha_{2, n_{2}} \beta_{1, n_{1}}=0 \text {. }
$$

But the last expression is zero if and only if there is a plane $H$, with

$$
I\left(P_{i}, C \cap H\right) \geq n_{i}+1 \quad \text { for } i=1,2 .
$$

This would contradict the definition of $r$, so the form does not vanish. 
(b) $r>0$. The form does not vanish since

(i) $k_{1} \neq k_{2}$,

(ii) $\alpha_{1, n_{1}}$ and $\alpha_{2, n_{2}}$ are nonzero, and

(iii) $\beta_{1, n_{1}+r}$ or $\beta_{2, n_{2}+r}$ is nonzero.

Hence the multiplicity of $i^{-1}\left(V_{4}^{1}\right)$ at $P_{1}+P_{2}$ is equal to the degree of the leading form of $R$ :

$$
\min \left(n_{1}+n_{2}-2+r, 2 n_{2}+r_{2}-1\right) .
$$

This gives the proposition.

Corollary 5.4. If a stationary secant $\overline{P_{1} P_{2}}$ is not a tangent to $C$ at any of the points $P_{1}, P_{2}$, then the multiplicity of $i^{-1}\left(V_{4}^{1}\right)$ at $P_{1}+P_{2}$ is

$$
r=\min \left(I\left(P_{1}, C \cap H\right), I\left(P_{2}, C \cap H\right)\right)-1,
$$

where $H$ is the plane spanned by the tangent lines to $C$ at $P_{1}$ and $P_{2}$.

The multiplicity of $i^{-1}\left(V_{4}^{1}\right)$ at $D=2 P$. Let $L$ be the tangent line to $C$ at the point $P$. Set $m_{2}=\max \left\{l \mid l P \in V_{l}^{l-2}\right\}$ or, equivalently, $m_{2}=I(P, C \cap H)$ for a general member $H$ of the pencil of planes containing $L$. If $P$ is not a flex on $C$, then $m_{2}=2$. Set $m_{3}=\max \left\{l \mid l P \in V_{l}^{l-3}\right\}$ or, equivalently, $m_{3}=\max _{H \supseteq L}\{I(P, C \cap H)\}$. Clearly $m_{3} \geq m_{2}+1$.

We now give our main result in the case $D=2 P$.

Proposition 5.5. The multiplicity of $i^{-1}\left(V_{4}^{1}\right)$ at $2 P$ is $\left[\left(m_{2}+m_{3}\right) / 2\right]-2$, where $[x]$ means the integral part of the real number $x$.

Proof. Let $t$ be a local parameter for $C$ at $P$. Without loss of generality we may assume that $C$ is parametrized locally at $P$ as

$$
\begin{aligned}
& X_{0}=1, \\
& X_{1}=t, \\
& X_{2}=\sum_{j \geq m_{2}} \alpha_{j} t^{j}, \quad \alpha_{m_{2}} \neq 0, \\
& X_{3}=\sum_{j \geq m_{3}} \beta_{j} t^{j}, \quad \beta_{m_{3}} \neq 0 .
\end{aligned}
$$

Let $s_{1}, s_{2}, s_{3}, s_{4}$ be local parameters for $C^{(4)}$ at $4 P$, where the $s_{k}$ are the $k$ th elementary functions in $t_{1}, t_{2}, t_{3}, t_{4}$; four replicas of $t$.

By Theorem I.4.2, we have

$$
\hat{O}_{V_{4}^{1}, 4 P}=K\left[\left[s_{1}, s_{2}, s_{3}, s_{4}\right]\right] /(\operatorname{det} M),
$$

where

$$
M=\left[\begin{array}{cccc}
1 & s_{1} & \sum_{j} \alpha_{j} W_{j}(\underline{s}) & \sum_{j} \beta_{j} W_{j}(s) \\
0 & 1 & \sum_{j} \alpha_{j} W_{j-1}(\underline{s}) & \sum_{j} \beta_{j} W_{j-1}(s) \\
0 & 0 & \sum_{j} \alpha_{j} W_{j-2}(\underline{s}) & \sum_{j} \beta_{j} W_{j-2}(s) \\
0 & 0 & \sum_{j} \alpha_{j} W_{j-3}(\underline{s}) & \sum_{j} \beta_{j} W_{j-3}(s)
\end{array}\right] .
$$


We see that

$$
\begin{aligned}
\operatorname{det} M= & \sum_{j \geq m_{2}} \alpha_{j} W_{j-2}(\underline{s}) \cdot \sum_{j \geq m_{3}} \beta_{j} W_{j-3}(\underline{s}) \\
& -\sum_{j \geq m_{2}} \alpha_{j} W_{j-3}(\underline{s}) \cdot \sum_{j \geq m_{3}} W_{j-2}(\underline{s}) .
\end{aligned}
$$

Let $S_{1}$ and $S_{2}$ be local parameters of $C^{(2)}$ at $2 P$, where the $S_{k}$ are the $k$ th symmetric functions in $T_{1}, T_{2}$; two formal replicas of $t$.

The map (5.1) induces a map

$$
i^{*}: K\left[\left[s_{1}, s_{2}, s_{3}, s_{4}\right]\right] \rightarrow K\left[\left[S_{1}, S_{2}\right]\right]
$$

Clearly $\hat{O}_{i^{-1}\left(V_{4}^{1}\right), 2 P} \cong K\left[\left[S_{1}, S_{2}\right]\right] /(R)$, where $R$ is the power series obtained by substituting $i^{*} s_{k}$ for $s_{k}$ in (5.2), for $k=1,2,3,4$. The multiplicity mult $_{2 P}\left(i^{-1}\left(V_{4}^{1}\right)\right)$ is the lowest value $e_{1}+e_{2}$ for any term $S_{1}^{e_{1}} S_{2}^{e_{2}}$ occurring in $R$. We will first find the $i^{*} s_{k}$. Let $s_{k}=s_{k}\left(t_{1}, t_{2}, t_{3}, t_{4}\right)$; that is: Regard $s_{k}$ as the $k$ th elementary symmetric function in four replicas of $t$ for $k=1, \ldots, 4$.

We define

$$
\psi_{k}\left(T_{1}, T_{2}\right)=s_{k}\left(T_{1}, T_{1}, T_{2}, T_{2}\right)
$$

Clearly $\psi_{k}\left(T_{1}, T_{2}\right)$ is symmetric in $T_{1}, T_{2}$ for $k=1, \ldots, k$. Hence there are unique functions $\phi_{k}\left(S_{1}, S_{2}\right)$ such that

$$
\phi_{k}\left(S_{1}\left(T_{1}, T_{2}\right), S_{2}\left(T_{1}, T_{2}\right)\right)=\psi_{k}\left(T_{1}, T_{2}\right)
$$

for $i=1, \ldots, k$.

One sees that $i^{*} s_{k}\left(S_{1}, S_{2}\right)=\phi_{k}\left(S_{1}, S_{2}\right)$ for all $k$.

We then obtain

$$
\begin{gathered}
i^{*} s_{1}=2 S_{1}, \quad i^{*} s_{2}=S_{1}^{2}+4 S_{2}, \\
i^{*} s_{3}=2 S_{1} S_{2}, \quad i^{*} s_{4}=S_{2}^{2} .
\end{gathered}
$$

We have

$$
\begin{aligned}
R= & \sum_{j \geq m_{2}} \alpha_{j}\left(i^{*} W_{j-2}\right) \cdot \sum_{j \geq m_{3}} \beta_{j}\left(i^{*} W_{j-3}\right) \\
& -\sum_{j \geq m_{2}} \alpha_{j}\left(i^{*} W_{j-3}\right) \cdot \sum_{j \geq m_{3}} \beta_{j}\left(i^{*} W_{j-2}\right),
\end{aligned}
$$

where $i^{*} W_{l}=W_{l}\left(i^{*} s_{1}, \ldots, i^{*} s_{4}\right)$ for all $l$.

We now must verify that the leading form of $R$ in the variables $S_{1}$ and $S_{2}$ is as stated in Proposition 5.5. This was done in detail in [Prpr]. We omit the very technical calculations here.

Corollary 5.6. If $P$ is not a flex on $C$, then the multiplicity of $i^{-1}\left(V_{4}^{1}\right)$ at $2 P$ is $\left[m_{3} / 2\right]-1$, where $m_{3}=I(P, C \cap H)$, for the osculating plane $H$ of $C$ at $P$. 
Comment 5.7. Assume:

(a) No plane intersects $C$ more than four times at any point;

(b) $C$ has no bitangents;

(c) $C$ has no flexes;

(d) no plane is osculating at more than one point of $C$;

(e) for each tangential trisecant line to $C$ tangent to $C$ at say $P_{1}$ and intersecting $C$ transversally at say $P_{2}$, the osculating plane at $P_{1}$ does not contain the tangent to $C$ at $P_{2}$.

Then it follows from Propositions 5.3 and 5.5 that the curve $i^{-1}\left(V_{4}^{1}\right)$ is nonsingular.

A nonsingular space curve has only finitely many tangential trisecants, flexes, bitangents, and hyperosculating or biosculating planes. Hence it follows that the curve (scheme) $i^{-1}\left(V_{4}^{1}\right)$ is always reduced.

This curve however, might, be reducible. As an example of this, take $C$ as the complete intersection of two quadric surfaces. Then $C$ is contained in four quadric cones, and each generatrix of each such cone is a stationary bisecant line. Hence $i^{-1}\left(V_{4}^{1}\right)$ has (at least) four components in this case.

A geometrical interpretation of the tangent cone $\mathscr{T}_{D}\left(i^{-1}\left(V_{4}^{1}\right)\right.$. In Definition 3.2 we described the (projectivized) tangent cone of a variety at a point. The tangent cone of the curve $i^{-1}\left(V_{4}^{1}\right)$ at a point $D$ is determined by the leading form of the relation $R$ as given in (5.5) in the case $D=2 P$, or as in (5.2) and (5.3) where the leading form is given explicitly in the case $D=P_{1}+P_{2}$, $P_{1} \neq P_{2}$.

In both cases the tangent cone is determined by a homogeneous polynomial of degree $m$ in two variables, where $m$ is the multiplicity of $i^{-1}\left(V_{4}^{1}\right)$ at $D$. This polynomial splits into $m$ linear factors. It turns out that in many cases each linear factor in the leading form corresponds to a point on the secant line $L$ with a certain geometrical significance. Clearly each linear factor corresponds to a point of the projectivized tangent cone $P \mathscr{T}_{D}\left(i^{-1}\left(V_{4}^{1}\right)\right)$. Hence we have an analogy to Result 4.2 in these cases. We would like to explain this more closely.

As usual we denote by $l(L)$ the point in the Grassmannian $G=G(1,3)$ corresponding to a line $L$. Set

$$
B=\{l(L) \mid L \text { satisfies }(\mathrm{a}) \text { or }(\mathrm{b}) \text { below }\} .
$$

(a) $L \cap C=\left\{P_{1}, P_{2}\right\}$, and $L$ is not a tangent line to $C$.

(b) $L \cap C=\{P\}$, and $L$ is a tangent, but not a flex tangent line to $C$ at $P$.

By the Trisecant Lemma the closure $\bar{B}$ is a surface in $G$. It is a standard fact that $\bar{B}$ is locally isomorphic to $C^{(2)}$ at points of $B$ under the map that sends the secant (tangent) line $l(L)$ to the divisor $P_{1}+P_{2}(2 P)$. Moreover $\bar{B}$ is nonsingular at points of $B$.

Let $S$ be the subcurve of $\bar{B}$ corresponding to stationary bisecants in the sense described earlier. Then $S$ is locally isomorphic to $i^{-1}\left(V_{4}^{1}\right)$ at points of $S \cap B$. 
Consider the Plücker embedding $G \subseteq P^{5}$. It is a well-known fact (see, for example, [GP, p. 16]) that the points of $S \cap B$ are exactly those points of $B$ such that the embedded tangent planes to $\bar{B}$ in $P^{5}$ are globally contained in $G$ (in fact as $\beta$-planes). For a point $l(L)$ on $S \cap B$, this tangent plane is $\check{H}$, where $H$ is the stationary plane in $P^{3}$ spanned by the divisor $2 D$ on $C$.

This information implies that if $C$ is not contained in a cone consisting of stationary bisecant lines, then the family of stationary bisecant lines envelope another curve $\mathscr{C}$ in $P^{3}$.

Considering the stationary bisecants as dual lines, the same family envelopes a curve [ in $\check{P}^{3}$.

The following is easily verified.

(1) $C$ is on a cone consisting of stationary bisecant lines $\Leftrightarrow A$ component of $\mathscr{C}$ degenerates to a point $\Leftrightarrow A$ component of [ is plane.

(2) $\mathscr{C}$ and [ are dual to each other, that is, [ parametrizes the osculating planes of $\mathscr{C}$, and vice versa.

(3) [ parametrizes the bitangent planes of $C$.

Since $i^{-1}\left(V_{4}^{1}\right)$ is locally isomorphic to $S$ at points of $S \cap B$, we can study the tangent cone to $S$ at $l(L)$ instead of that of $i^{-1}\left(V_{4}^{1}\right)$ at $D$. Since the embedded tangent space of $B$ at $l(L)$ is the dual plane $\check{H}$, we can embed $\mathscr{T}_{l(L)}(S)$ as a union of $m$ lines in $\check{H}$ through the point $l(L)$. But a line in $\check{H} \subset G$ through $l(L)$ corresponds to a pencil of lines in $H \subset P^{3}$ through some point $Q$ of $L$. Such points $Q$ of $L$ are exactly the points of $L \cap \mathscr{C}$ arising from the local branch(es) of $S$. This means that the explicit calculations of the leading forms performed earlier in $\S 5$ tell us how the points of $L \cap \mathscr{C}$ are located in Cases a and $b$.

Case a. $L \cap C=\left\{P_{1}, P_{2}\right\}, L$ is not a tangent line. Set

$$
r=\min \left(I\left(P_{1}, C \cap H\right), I\left(P_{2}, C \cap H\right)\right)-1
$$

for the stationary plane $H$. By (5.2) the leading form in $t_{1}, t_{2}$ is (up to a constant)

$$
\alpha_{1,1} \beta_{2, r+1} t_{2}^{r}-\alpha_{2,1} \beta_{1, r+1} t_{1}^{r} \text {. }
$$

Hence the multiplicity $m$ is $r$, and we get $r$ distinct points of $L \cap \mathscr{C}$ outside $C$ unless either $\beta_{1, r+1}$ or $\beta_{2, r+1}$ is zero. If, say, $\beta_{1, r+1}=0$, which means $I\left(P_{1}, C \cap H\right) \geq r+1$, then all $r$ points of $L \cap \mathscr{C}$ collapse to one point. It turns out that this single point is $P_{2}$. See Result 5.8. below, or Remark 5.9.

Case b. $L \cap C=\{P\}, L$ is tangent to $C$ at $P$, but $P$ is not a flex. We recall the definition $m_{3}=I(P, C \cap H)$, where $H$ is the osculating (stationary) plane of $C$ at $P$.

We recall that the leading form in $S_{1}, S_{2}$ is $S_{2}^{\left(m_{3}-3\right) / 2}$ when $m_{3}$ is odd and $\left(S_{1}+k S_{2}\right) S^{m_{3} / 2-2}$ when $m_{3}$ is even. It turns out that the factor $S_{2}$ corresponds to the (secant) point $P$ of $C \cap L$, while the factor $S_{1}+k S_{2}$ corresponds to a point outside $P$. "In general," when $m_{3}=4$, we get only the last factor. 
In Cases a and b, we have another description of the points of $L \cap \mathscr{C}$ arising from the local branch(es) of $S$. Denote by $m$ the multiplicity of $S$ at $l(L)$.

Result 5.8. $Q \in L$ is a point of $\mathscr{C}$ iff there exists a cone $N$ of degree $m+1$ with vertex at $Q$ such that $\operatorname{Sing}(N) \not \supset L$ and:

Case a. $I\left(P_{i}, C \cap N\right) \geq m+2$ for $i=1,2$.

Case b. $I(P, C \cap N) \geq 2 m+4$.

Idea of proof. Let $F$ be the surface in $P^{3}$ swept out by the stationary bisecant lines. Let $C^{\prime}$ be a dummy curve on $F$ transversal to the ruling around $L$. Regard $L$ as a singular trisecant to $C \cup C^{\prime}$. The point $l(L)$ is contained in a nonreduced component of the trisecant curve in $G$. Then apply Result 4.3 in the case $n=3$.

Remark 5.9. Recall the local parametrizations of $C$ introduced in the proof of Proposition 5.3. Referring to these parametrizations, Result 5.8 translates in Case a to $Q=(1, k, 0,0)$ is a point on $L \cap \mathscr{C}$ iff

$$
\left(\frac{k_{2}-k}{k_{1}-k}\right)^{r}=\frac{\beta_{1, r+1}}{\beta_{2, r+1}} \cdot \frac{\alpha_{2,1}^{r+1}}{\alpha_{1,1}^{r+1}} .
$$

A similar result can be obtained in Case $b$.

We might return to a more detailed study of the curves $\mathscr{C}, S$, [ in another paper. With the information we have now it is easy to compute the "expected" genera, degrees, and numbers of cusps of these curves.

\section{REFERENCES}

[ACGH] E. Arbarello, M. Cornalba, P. A. Griffiths and J. Harris, Geometry of algebraic curves, Vol. I, Springer-Verlag, Berlin, Heidelberg, and New York, 1985.

[G] M. Gonzalez, Singularités de la courbe des bisécantes stationnaires, C. R. Acad. Sci. Paris Ser. I 305 (1987), 341-343.

[GP] L. Gruson and C. Peskine, Courbes de l'espace projectif, variétés de sécantes, Enumerative Geometry and Classical Algebraic Geometry, Progress in Math., vol. 24, Birkhäuser, 1982, pp. $1-31$.

[J] T. Johnsen, The singularities of the 3-secant curve associated to a space curve, Trans. Amer. Math. Soc. 295, (1986), 107-118.

[Prpr] __, Local properties of secant varieties in symmetric products, Preprint No. 1, 1987, Univ. of Oslo.

Institute of Mathematical Sciences, University of Tromsø, P. O. Box 953, 9001 TROMSø, NORWAY 